\title{
ON SPECTRALITY OF THE ALGEBRA OF CONVOLUTION DOMINATED OPERATORS
}

\author{
GERO FENDLER \\ Finstertal 16, D-69514 Laudenbach, Germany \\ E-mail: gero.fendler@t-online.de \\ KARLHEINZ GRÖCHENIG \\ Fakultät für Mathematik, Universität Wien \\ Nordbergstrasse 15, A-1090 Wien, Austria \\ E-mail: karlheinz.groechenig@univie.ac.at \\ MICHAEL LEINERT \\ Institut für Angewandte Mathematik, Fakultät für Mathematik \\ Im Neuenheimer Feld 288, D-69120 Heidelberg, Germany \\ E-mail: leinert@math.uni-heidelberg.de
}

\begin{abstract}
If $G$ is a discrete group, the algebra $C D(G)$ of convolution dominated operators on $l^{2}(G)$ (see Definition 1 below) is canonically isomorphic to a twisted $L^{1}$-algebra $l^{1}\left(G, l^{\infty}(G), T\right)$. For amenable and rigidly symmetric $G$ we use this to show that any element of this algebra is invertible in the algebra itself if and only if it is invertible as a bounded operator on $l^{2}(G)$, i.e. $C D(G)$ is spectral in the algebra of all bounded operators. For $G$ commutative, this result is known (see [1], [6]), for $G$ noncommutative discrete it appears to be new. This note is about work in progress. Complete details and more will be given in [3].
\end{abstract}

Let $G$ be a discrete group. For $x \in G$ we denote by $\lambda(x)$ the operator of left translation on $l^{1}(G)$ and on $l^{2}(G)$, i.e. $\lambda(x) f(y)=f\left(x^{-1} y\right)$ for $f \in l^{1}(G)$ or $f \in l^{2}(G), x, y \in G$. By $B\left(l^{2}(G)\right)$ we denote the algebra of bounded operators on $l^{2}(G)$.

For an operator $A: l^{2}(G) \rightarrow l^{2}(G)$ let $A(x, y)=\left\langle A \delta_{y}, \delta_{x}\right\rangle, x, y \in G$ be its matrix, where by $\delta_{z}$ we denote the characteristic function of the one point set $\{z\} \subset G$, and $\langle$, is the usual scalar product of the Hilbert space $l^{2}(G)$.

2000 Mathematics Subject Classification: Primary 47B35; Secondary 43A20.

Key words and phrases: convolution dominated operators, inverse-closed subalgebras, symmetry.

Karlheinz Gröchenig was supported by Marie-Curie Excellence Grant MEXT-CT 2004517154. 
Definition 1. The operator $A$ is called convolution dominated if there exists some $a \in$ $l^{1}(G)$ such that

$$
|A(x, y)| \leq a\left(x y^{-1}\right), \quad \forall x, y \in G
$$

We define its norm as

$$
\|A\|_{1}:=\inf \left\{\|a\|_{l^{1}}: a \in l^{1}(G),|A(x, y)| \leq a\left(x y^{-1}\right) \forall x, y \in G\right\} .
$$

By $C D(G)$ we denote the Banach space of all convolution dominated operators.

We remark that $A \in B\left(l^{2}(G)\right)$ is convolution dominated if the supremum norms of the side diagonals of its matrix are summable, i.e. if

$$
\sum_{z \in G} \sup _{\left\{x, y: x y^{-1}=z\right\}}|A(x, y)|<\infty .
$$

Moreover this quantity just equals the norm $\|A\|_{1}$.

Since $l^{1}(G)$ is a convolution algebra it follows that the space of convolution dominated operators is an algebra under composition of operators. Moreover it is not hard to see that it becomes a Banach $*$-algebra (containing an identity) with respect to the usual involution of operators in $B\left(l^{2}(G)\right)$.

We consider $l^{\infty}(G)$ as a $C^{*}$-algebra (really, it is a von Neumann algebra) with respect to pointwise multiplication and complex conjugation as involution. For $x \in G$ let $T_{x}: l^{\infty}(G) \rightarrow l^{\infty}(G)$ denote the $C^{*}$-automorphism of the algebra $l^{\infty}(G)$ given by left translation $T_{x} n(z)=n\left(x^{-1} z\right), n \in l^{\infty}(G)$, so that $x \mapsto T_{x}$ is a representation of $G$ in the group of $C^{*}$-automorphisms of $l^{\infty}(G)$. With these data we form the twisted $L^{1}$-algebra in the sense of Leptin $[7,8,9]$ (with trivial factor system), which we denote by $l^{1}\left(G, l^{\infty}(G), T\right)$, or simply by $\mathcal{L}$. It consists of all functions $f: G \rightarrow l^{\infty}(G)$, such that $\|f\|:=\sum_{z \in G}\|f(z)\|_{\infty}<\infty$. The product (twisted convolution) is given by

$$
h \star f(x)=\sum_{y} T_{y} h(x y) f\left(y^{-1}\right), \text { for } x \in G, h, f \in \mathcal{L}
$$

and the involution by

$$
h^{*}(x)=\overline{T_{x^{-1}} h\left(x^{-1}\right)}, \text { for } x \in G, h \in \mathcal{L} .
$$

The $C^{*}$-algebra $l^{\infty}(G)$ is isometrically represented as multiplication operators on $l^{2}(G)$ :

$$
D^{m} f(x)=m(x) f(x) \text {, where } x \in G, f \in l^{2}(G), m \in l^{\infty}(G) .
$$

We have the covariance relation $\lambda\left(x^{-1}\right) D^{m} \lambda(x)=D^{T_{x}^{-1} m}$, so from $\lambda: G \rightarrow B\left(l^{2}(G)\right)$ and $D: l^{\infty}(G) \rightarrow B\left(l^{2}(G)\right)$ we obtain a representation $R$ of $\mathcal{L}$ on $l^{2}(G)$. An element $f \in \mathcal{L}$ may be uniquely written as

$$
f=\sum_{z \in G} m_{z} \delta_{z},
$$

where $m_{z}=f(z) \in l^{\infty}(G)$. The representation $R: l^{1}\left(G, l^{\infty}(G), T\right) \rightarrow B\left(l^{2}(G)\right)$ is given by the prescription

$$
R f=\sum_{z} \lambda(z) D^{m_{z}} .
$$


This means that we read $f$ as an operator on $l^{2}(G)$ by placing $f(z)$ (which is an $l^{\infty}$ function) on the $z$ th side-diagonal of the matrix defining the operator $R f$. To be more precise

$$
(R f)(x, y)=f\left(x y^{-1}\right)(y), \quad x, y \in G,
$$

i.e. the entry at position $(x, y)$, which lies on the $z$ th side-diagonal $\left(z=x y^{-1}\right)$, is given by $f(z)(y)=m_{z}(y)$. We call $R$ the canonical representation of $\mathcal{L}$.

Proposition 2. The map $R: l^{1}\left(G, l^{\infty}(G), T\right) \rightarrow C D(G)$ is an isometric $*$-isomorphism.

Recall that a Banach algebra $A$ with involution is called symmetric if every positive element has its spectrum contained in the non-negative reals, i. e. $\operatorname{sp}\left(a^{*} a\right) \subset[0, \infty)$ $\forall a \in A$. Accordingly, a locally compact group $G$ is called symmetric if its convolution algebra $L^{1}(G)$ is symmetric. Various classes of groups are known to be symmetric, e. g. Abelian locally compact groups, compact groups, finite extensions of discrete nilpotent groups, compactly generated groups of polynomial growth.

Leptin and Poguntke [10] showed that the groups of the first three classes satisfy the stronger property of rigid symmetry. Namely for any $C^{*}$-algebra $C$ the projective tensor product $L^{1}(G) \hat{\otimes} C$ is symmetric. Later Poguntke [11] showed that all nilpotent locally compact groups are rigidly symmetric.

Define a map

$$
Q: l^{1}\left(G, l^{\infty}(G), T\right) \rightarrow l^{1}(G) \hat{\otimes} B\left(l^{2}(G)\right)
$$

by

$$
f=\sum_{v} m_{v} \delta_{v} \mapsto \sum_{v} \delta_{v} \otimes \lambda(v) D^{m_{v}} .
$$

Proposition 3. The above defined map $Q$ is an isometric $*$-isomorphism of $\mathcal{L}$ onto a closed subalgebra of $l^{1}(G) \hat{\otimes} B\left(l^{2}(G)\right)$.

Since symmetry passes to closed subalgebras, we have

COROLlary 4. Let $G$ be a discrete rigidly symmetric group, then $\mathcal{L}$ and $C D(G)$ are symmetric Banach *-algebras.

Recall that by $D: m \mapsto D^{m}$ the $C^{*}$-algebra $l^{\infty}(G)$ is faithfully represented by multiplication operators on $l^{2}(G)$. On the Hilbert space $l^{2}\left(G, l^{2}(G)\right)$, the $D$-regular representation $\lambda^{D}$ of $\mathcal{L}=l^{1}\left(G, l^{\infty}(G), T\right)$ is defined (see $[9, \S 3]$ ) by

$$
\lambda^{D}(f) \xi(x)=\sum_{y} D^{T_{y} f(x y)} \xi\left(y^{-1}\right), \quad \text { where } \xi \in l^{2}\left(G, l^{2}(G)\right), f \in \mathcal{L} .
$$

On the other hand $R: \mathcal{L} \rightarrow C D(G) \subset B\left(l^{2}(G)\right)$ is a *-representation of $\mathcal{L}$ on $l^{2}(G)$. We identify $l^{2}\left(G, l^{2}(G)\right)$ with $l^{2}(G \times G)$ and define a multiple of the canonical representation by letting the operators $R(f)=\sum_{y} \lambda(y) D^{f(y)}, f \in \mathcal{L}$, act in the first coordinate of the $l^{2}(G \times G)$-functions only. The unitary operator $S \xi(x, z)=\xi(x z, z)$, where $\xi \in l^{2}(G \times G)$ actually intertwines these two representations, so we have

Proposition 5. The D-regular representation of $\mathcal{L}$ is equivalent to a multiple of the canonical representation. 
These representations are faithful $*$-representations of $\mathcal{L}$. Hence the greatest $C^{*}$ seminorm

$$
\|f\|_{*}=\sup \{\|\pi(f)\|: \pi \text { a } * \text {-representation of } \mathcal{L} \text { on a Hilbert space }\}, \quad f \in \mathcal{L},
$$

is in fact a norm.

Corollary 6. Let $G$ be an amenable discrete group, then the greatest $C^{*}$ semi-norm on $\mathcal{L}$ equals the operator norm on $C D(G)$.

Proof. It follows from [9, Satz 6] of Leptin that for the representation $D$ of $l^{\infty}(G)$ the $D$-regular representation $\lambda^{D}$ defines the greatest $C^{*}$ semi-norm on $\mathcal{L}$. Denoting this norm by $\|.\|_{*}$ we have for $f \in \mathcal{L}$ :

$$
\|f\|_{*}=\left\|\lambda^{D}(f)\right\|=\|R(f)\|_{B\left(l^{2}(G)\right)},
$$

where the last equality follows from Proposition 5.

For an element $a$ of a normed algebra $A$ we denote by $r_{A}(a)$ its spectral radius.

Proposition 7. Let $G$ be a discrete, amenable, and rigidly symmetric group. Then for $f \in \mathcal{L}$

$$
r_{\mathcal{L}}\left(f^{*} f\right)=r_{C D(G)}\left(R(f)^{*} R f\right)=\|R(f)\|_{B\left(l^{2}(G)\right)}^{2} .
$$

Proof. By Corollary 4 we know that $\mathcal{L}$ and $C D(G)$ are symmetric. By a theorem of Pták [12] it follows that $\|f\|_{*}^{2}=r_{\mathcal{L}}\left(f^{*} f\right)=r_{C D(G)}\left(R(f)^{*} R(f)\right)$ (see e.g. [2, §41 Corollary 8]). Corollary 6 now proves the assertion.

THEOREM 8. Let $G$ be a discrete, amenable, and rigidly symmetric group. If $f \in \mathcal{L}$ is such that $R(f) \in C D(G)$ has an inverse in $B\left(l^{2}(G)\right)$ then $f^{-1}$ exists in $\mathcal{L}$ and $R\left(f^{-1}\right)=$ $R(f)^{-1}$ is in $C D(G)$.

Proof. If $f \in \mathcal{L}$ is hermitian, i.e. $f=f^{*}$, then

$$
r_{\mathcal{L}}(f)^{2}=r_{\mathcal{L}}\left(f^{*} f\right)=\|R(f)\|_{B\left(l^{2}(G)\right)}^{2} .
$$

We apply Hulanicki's Lemma [5, Prop. 2.5] and obtain that

$$
s p_{\mathcal{L}}(f)=s p_{B\left(l^{2}(G)\right)}(R(f)), \quad \forall f=f^{*} \in \mathcal{L} .
$$

This implies

(see Lemma $[4,3.7])$.

$$
s p_{\mathcal{L}}(f)=s p_{B\left(l^{2}(G)\right)}(R(f)), \quad \forall f \in \mathcal{L},
$$

\section{References}

[1] A. G. Baskakov, Abstract harmonic analysis and asymptotic estimates for elements of inverse matrices, Mat. Zametki 55 (1992), 17-26, 155.

[2] F. Bonsall and J. Duncan, Complete Normed Algebras, Springer Verlag, 1973.

[3] G. Fendler, K. Gröchenig, M. Leinert, Convolution-dominated operators on discrete groups, in preparation.

[4] G. Fendler, K. Gröchenig, M. Leinert, J. Ludwig, and C. Molitor-Braun, Weighted group algebras on groups of polynomial growth, Math. Z. 245 (2003), 791-821. 
[5] A. Hulanicki, On the spectrum of convolution operators on groups with polynomial growth, Invent. Math. 17 (1972), 135-142.

[6] V. Kurbatov, Functional Differential Operators and Equations, Math. Appl. 473, Kluwer, 1999.

[7] H. Leptin, Verallgemeinerte $L^{1}$-Algebren und projektive Darstellungen lokal kompakter Gruppen I, Invent. Math. 3 (1967), 257-281.

[8] H. Leptin, Verallgemeinerte $L^{1}$-Algebren und projektive Darstellungen lokal kompakter Gruppen II, Invent. Math. 4 (1967), 68-86.

[9] H. Leptin, Darstellungen verallgemeinerter $L^{1}$-Algebren, Invent. Math. 5 (1968), 192-215.

[10] H. Leptin and D. Poguntke, Symmetry and non-symmetry for locally compact groups, J. Funct. Anal. 33 (1979), 119-134.

[11] D. Poguntke, Rigidly symmetric $L^{1}$-group algebras, Seminar Sophus Lie 2 (1992), 189-197.

[12] V. Pták, On the spectral radius in Banach algebras with involution, Bull. London Math. Soc. 2 (1970), 327-334. 
